REVISTA PORTUGUESA DE $P S \bigcirc \bigcirc \bigcirc$

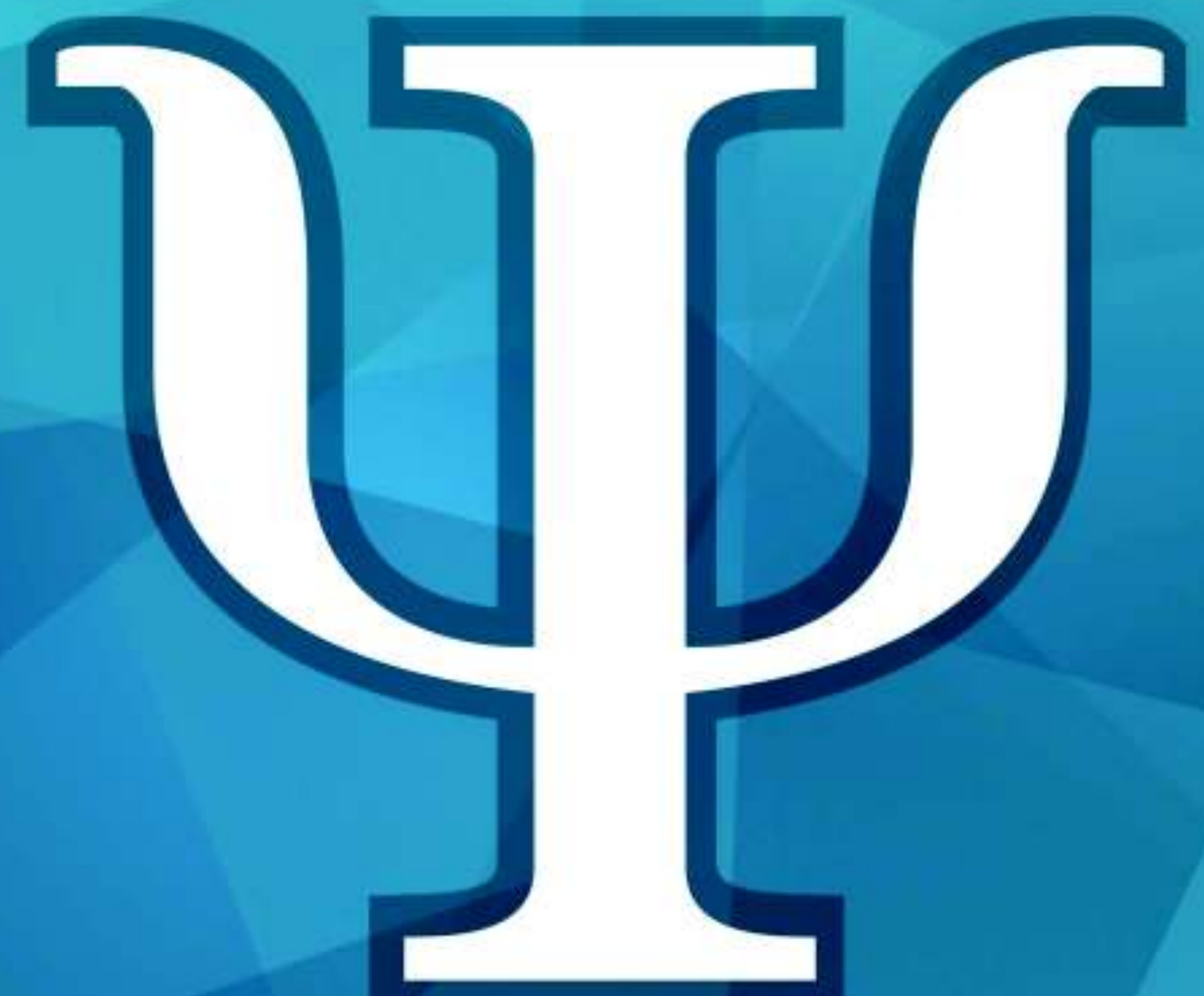

$\Psi$ 


\section{Conselho Editorial / Editorial Board}

Editor:

João Manuel Moreira

Faculdade de Psicologia, Universidade de Lisboa

\section{Conselho Editorial / Editorial Board:}

Adelina Lopes da Silva

Faculdade de Psicologia, Universidade de Lisboa

Alexandra Reis

Faculdade de Ciências Humanas e Sociais, Universidade do Algarve

Amâncio da Costa Pinto

Faculdade de Psicologia e CE, Universidade do Porto

Ana Margarida Veiga Simão

Faculdade de Psicologia, Universidade de Lisboa

Bárbara Figueiredo

Escola de Psicologia, Universidade do Minho

Danilo Silva

Faculdade de Psicologia, Universidade de Lisboa

Félix Neto

Faculdade de Psicologia e CE, Universidade do Porto

Helio Carpinteiro

Universidade Complutense de Madrid

Isabel Sá

Faculdade de Psicologia, Universidade de Lisboa

Isabel Soares

Escola de Psicologia, Universidade do Minho

Jean Guichard

Conservatoire National des Arts et Métiers

José Frederico Marques

Faculdade de Psicologia, Universidade de Lisboa

José H. Ferreira-Marques

Faculdade de Psicologia, Universidade de Lisboa

José Keating

Escola de Psicologia, Universidade do Minho

José Tomás da Silva

Faculdade de Psicologia e CE, Universidade de Coimbra

Leonel Garcia-Marques

Faculdade de Psicologia, Universidade de Lisboa

Leonor Cardoso

Faculdade de Psicologia e CE, Universidade de Coimbra

Luísa Barros

Faculdade de Psicologia, Universidade de Lisboa

Luísa Morgado

Faculdade de Psicologia e CE, Universidade de Coimbra

Manuel Rafael

Faculdade de Psicologia, Universidade de Lisboa
Maria do Céu Taveira

Escola de Psicologia, Universidade do Minho

Maria Eduarda Duarte

Faculdade de Psicologia, Universidade de Lisboa

Maria José Chambel

Faculdade de Psicologia, Universidade de Lisboa

Mário Ferreira

Faculdade de Psicologia, Universidade de Lisboa

Mário Simões

Faculdade de Psicologia e CE, Universidade de Coimbra

Mark Savickas

Northeast Ohio Medical University

Paulo Ventura

Faculdade de Psicologia, Universidade de Lisboa

Pedro B. Albuquerque

Escola de Psicologia, Universidade do Minho

Rocío Fernandez-Ballesteros

Universidad Autónoma de Madrid

Rosário Lima

Faculdade de Psicologia, Universidade de Lisboa

Salomé Vieira Santos

Faculdade de Psicologia, Universidade de Lisboa

São Luís Castro

Faculdade de Psicologia e CE, Universidade do Porto

Assistente Editorial / Editorial Assistant:

Rita Monteiro

Editores e Diretores Eméritos / Emeriti Editors and Directors:

Henrique Barahona Fernandes

Director, 1967-1991

Agostinho Pereira

Editor, 1967-1969

José Luiz Simões da Fonseca

Editor, 1969-1971

José H. Ferreira-Marques

1992-2001

Danilo Silva

2002-2005

Manuel Rafael

2006-2011

\section{Instruções aos autores:}

https://sites.google.com/site/revistaportuguesadepsicologia /instrucoes-aos-autores

ISSN 2183-3699 (versão electrónica / electronic version) ISSN 0872-0304 (versão impressa / print version) 


\title{
Sintomatologia depressiva em estudantes universitários portugueses
}

\author{
Rui C. Campos \\ Departamento de Psicologia, Escola de Ciências Sociais e \\ Centro de Investigação em Educação e Psicologia, Universidade de Évora
}

\section{RESUMO}

Com este trabalho pretendeu-se contribuir para caracterizar a população universitária portuguesa no que respeita à presença de sintomatologia depressiva. A versão portuguesa do Inventário de Depressão de Beck-II (BDI-II) e uma ficha de dados demográficos foram aplicadas a uma amostra de 547 estudantes universitários de diversas instituições do ensino superior portuguesas. O sintoma depressivo mais relatado pelos estudantes foi Alterações nos hábitos de sono, destacando-se também: Sentimentos de Culpa, Autocrítica, Choro (apenas nas raparigas), Agitação, Indecisão, Perda de Energia (apenas nas raparigas), Alterações no Apetite, Dificuldades de concentração e Cansaço ou fadiga. A sintomatologia depressiva foi significativamente mais intensa nos estudantes do sexo feminino relativamente aos do sexo masculino, embora a magnitude do efeito da diferença seja relativamente pequena. Das variáveis sociodemográficas estudadas, quatro apresentam uma relação significativa com o BDI-II: Grau de satisfação com o curso, Já foi ao psicólogo, Foi ao psicólogo com uma certa regularidade e Número de vezes que foi ao médico no último ano.

Palavras-chave: Sintomas depressivos; Variáveis sociodemográficas; Estudantes universitários portugueses

\begin{abstract}
This work aimed to give a contribution to the characterization of the Portuguese university population regarding depressive symptoms. The Portuguese version of the Beck Depression Inventory-II (BDI-II) and a sociodemographic questionnaire were administered to a sample of 547 university students from several Portuguese university institutions. The most prevalent symptom was Changes in sleeping pattern. Also important were Guilty feelings, Self-criticalness, Crying (only in female students), Agitation, Indecisiveness, Loss of energy (only in female students), Changes in appetite, Concentration difficulty, and Tiredness or fatigue. Depressive symptoms were significantly more severe in female students relatively to male students, although the magnitude of the effect is relatively small. Four socio-demographic variables presented a significant relation with the BDI-II: Satisfaction with the course, Went to psychologist, Went to psychologist regularly, and Number of times went to the doctor last year.
\end{abstract}

Keywords: Depressive symptoms; Socio-demographic variables; Portuguese university students

\section{INTRODUÇÃO}

O estudo da prevalência de sintomas psicopatológicos em diferentes populações revela-se de grande importância, uma vez que pode ajudar os clínicos na organização dos serviços de apoio à comunidade e na implementação de práticas de intervenção específicas destinadas ao tratamento desses sintomas. Além disto, são também de grande importância pelas implicações que podem ter ao nível da prevenção.

Segundo a Organização Mundial de Saúde (OMS, 2012) a depressão é a principal causa de incapacidade no mundo inteiro, constituindo atualmente um importante problema de saúde pública (OMS, 2015). Segundo dados 